\title{
Phytochimiques des plantes médicinales utilisées dans la prise en charge des maladies infantiles au Sud- Bénin
}

\author{
Sabine Aribikè Kouchadé \\ Laboratoire de Botanique et Ecologie Végétale (LaBEV), \\ Faculté des Sciences et Techniques (FAST), \\ Université d’Abomey-Calavi, Cotonou, Bénin \\ Arlette Raymonde Adjatin \\ Laboratoire de Biotechnologie, Ressources Génétiques et Amélioration des \\ Espèces Animales et Végétales (BIORAVE), \\ Faculté des Sciences et Techniques (FAST) de Dassa, \\ Université d’Abomey-Calavi, Cotonou, Bénin \\ Aristide Cossi. Adomou \\ Hospice Gbèwonmèdéa Dassou \\ Akpovi Akoègninou \\ Laboratoire de Botanique et Ecologie Végétale (LaBEV), \\ Faculté des Sciences et Techniques (FAST), \\ Université d'Abomey-Calavi, Cotonou, Bénin
}

doi: 10.19044/esj.2016.v13n3p471 URL:http://dx.doi.org/10.19044/esj.2016.v13n3p471

\begin{abstract}
In southern Benin, medicinal plants are used for the health care of children aged 0 to 12 years. The present study aims to highlight some medicinal plants used in southern Benin to treat childhood diseases including malaria, candidiasis, teething disorders, infant colic and diarrhea. The phytochemical screening of the leafy stems of the selected plants revealed the presence of alkaloids, tannins (gallic and catechic), coumarins, anthocyanins and leuco-anthocyans, quinone derivatives, triterpenoids, steroids, saponosides and reducing sugars. These various metabolites possess various therapeutic properties involved in the treatment of the indicated disorders. This would confirm the therapeutic uses reported by the populations surveyed. However, the evaluation of antimalarial, antibacterial, anti-inflammatory and analgesic activities as well as the toxicity test of the aqueous extract of these plants would be necessary.
\end{abstract}


Keywords: Phytochemical screening, medicinal plants, infantile diseases, therapeutic property, South Benin.

\section{Résumé}

Au sud du Bénin, des plantes médicinales sont utilisées pour les soins de santé des enfants de 0 à 12 ans. La présente étude se propose de valoriser quelques plantes médicinales utilisées au sud du Bénin pour traiter les maladies infantiles dont le paludisme, la candidose, les troubles de dentition, la colique du nourrisson et la diarrhée. Le criblage phytochimique des rameaux feuillés des 10 plantes retenues révèle la présence d’alcaloïdes, de tanins (gallique et catéchique), de coumarines, d’anthocyanes et de leucoanthocyanes, de dérivés quinones, de triterpénoïdes, de stéroïdes, de saponosides et de sucres réducteurs. Ces divers métabolites possèdent diverses propriétés thérapeutiques pouvant intervenir dans le traitement des troubles indiqués. Ce qui confirmerait l'usage thérapeutique signalé par les populations enquêtées. Cependant l'évaluation des activités antipaludique, antibactérienne, anti-inflammatoire, analgésique de même que le test de toxicité de l'extrait aqueux de ces plantes seraient nécessaires.

Mots-clefs : Criblage phytochimique, plantes médicinales, maladies infantiles, propriété thérapeutique, Sud-Bénin.

\section{Introduction}

L’usage des plantes à des fins thérapeutiques est rapporté dans les littératures antique arabe, chinoise, égyptienne, hindou, grecque, romaine (Anonyme, 1974). Malgré les progrès de la biologie et de la médecine de l'heure, la majorité des populations des pays en voie de développement n’ont pas accès aux soins de santé suffisants à cause de leurs faibles revenus économiques (Singh \& Singh, 2012 ; OMS, 2012). A cela, s’ajoutent d'autres facteurs dont le manque et la mauvaise répartition des infrastructures sanitaires, la faible couverture des régions en médecins.

En effet, au Bénin, on recense plus de 7500 tradipraticiens répartis dans l'ensemble du territoire national (MS/PNPMT, 2009), contre seulement 600 médecins pour près de 10 millions d'habitants (SNIGS-MS, 2014). Bon nombre de ces agents de santé sont concentrés dans les zones urbaines, ce qui complique l'accessibilité géographique à la médecine moderne par les populations des zones rurales (Zerbo et al, 2007 ; Dresse et al, 2013). On note également le faible nombre de pédiatres (17 pédiatres pour une population de 5369774 habitants; INSAE, 2013) au sud du Bénin, or la norme exigée par l'OMS est de 25 personnels qualifiés pour 10000 habitants. 
Face à ces contraintes, les ressources végétales occupent une grande place dans la vie des populations (Mangambu et al., 2008). Les statistiques de l’Organisation Mondiale de la Santé (OMS, 2002), encore en vigueur selon Mangambu (2013) montrent que plus de $80 \%$ des populations africaines ont recours à la médecine et à la pharmacopée traditionnelle pour faire face aux problèmes de santé. Cet intérêt pour l'ethnomédecine vient de la disponibilité et de l'accessibilité des ressources végétales médicinales dans les pays en voie de développement. En effet, les estimations de Kolling, (2010) et de Mangambu et al. (2012) ont révélé la présence de plus de 200.000 espèces de plantes dans ces pays sur les 300.000 que compte la planète.

En Afrique, le pouvoir thérapeutique des plantes était connu des ancêtres et des parents de façon empirique (N'Guessan et al. 2009) mais, la composition chimique des médicaments utilisés tous les jours par ces nombreuses populations, pour leurs soins de santé était ignorée. En effet, pour la plupart de ces plantes, les composés chimiques responsables des activités biologiques signalées ainsi que leur toxicité restent inconnus (Agbankpè et al., 2015).

Au Bénin comme dans d’autres pays en voie de développement, plusieurs études scientifiques (Adjanohoun, 1989; Adomou et al., 2012, Dassou et al., 2015 ; Kouchadé et al., 2016 ; etc.) se sont consacrées à l'inventaire ethnobotanique afin de contribuer à la connaissance des plantes médicinales. Selon Vandaelen (2002), l'exploitation de l'héritage phytothérapeutique ne peut demeurer statique et se limiter à la seule collecte de recettes traditionnelles. Pour les valoriser, il est nécessaire d'effectuer des recherches phytochimiques, pharmacologiques et cliniques. Car, la méconnaissance des composés chimiques responsables d'activités biologiques des plantes utilisées cause d’énormes préjudices aux prescripteurs, à la base d'irrationalisme des doses conduisant au surdosage, au sous dosage, aux intoxications et voir même à la mort (Mangambu et al., 2014). Aussi, les études des propriétés biologiques et chimiques ont montré que la flore béninoise a un réel potentiel thérapeutique pouvant servir à traiter ou prévenir de nombreuses maladies. C'est le cas des travaux sur des plantes qui ont montré des propriétés antipaludiques (Lagnika, 2005 ; Shuaibu et al., 2008); propriétés galactogènes et emménagogues (Deleke et al., 2011); propriétés d'alicaments (Adjatin et al., 2013); propriétés antibactériennes (Agbankpé et al., 2015) et antifongique (Tra Bi et al., 2008; Soro et al., 2010).

C'est dans le souci d'apporter de nouvelles données sur les plantes utilisées dans les soins de santé des enfants de 0 à 12 ans que cette étude a été réalisée. Son objectif général est de valoriser les plantes médicinales 
utilisées au sud du Bénin pour traiter les maladies infantiles. De façon spécifique, il s’agit de:

- sélectionner les principales plantes utilisées pour le traitement des maladies infantiles au sud du Bénin ;

- identifier les métabolites secondaires contenus dans les principales plantes impliquées dans le traitement des maladies infantiles au sud du Bénin.

\section{Matériel et méthodes}

\section{Milieu d'étude}

Les études ethnobotaniques ont été conduites à travers tout le sud du Bénin qui s'étend entre les latitudes $6^{\circ} 25 \mathrm{~N}$ et $7^{\circ} 30 \mathrm{~N}$ et les longitudes $1^{\circ} 35 \mathrm{E}$ et $2^{\circ} 49 \mathrm{E}$ et couvre une superficie de $17109 \mathrm{~km}^{2}$. Le climat est de type subéquatorial, caractérisé par un régime pluviométrique bimodal avec deux saisons pluvieuses alternées par deux saisons sèches. La température moyenne annuelle est de $28^{\circ} \mathrm{C}$ et l'humidité de l'air varie entre $69 \%$ et $97 \%$ (Akoègninou, 2004). Les sols les plus dominants sont : les sols ferralitiques sur sédiments argileux, les sols hydromorphes dans les vallées, les bas-fonds et les plaines alluviales, les vertisols dans la dépression de la Lama et les sols bruns eutrophes tropicaux (Igué et al., 2013).

Sur le plan phytogéographique, le sud du Bénin compte 1170 espèces végétales, elle appartient à la zone guinéo-congolaise. La végétation est une mosaïque d'îlots de forêts denses humides, de savanes, de prairies, de mangrove et de jachères (Adomou et al., 2011). La population est de 5369 774 habitants avec une densité qui varie entre 100 habitants $/ \mathrm{km}^{2}$ en général à 322 habitants $/ \mathrm{km}^{2}$ dans le département de l’Atlantique (INSAE, 2013).

\section{Matériel}

Le matériel végétal est constitué de rameaux feuillés issus de dix espèces végétales les plus utilisées pour soigner cinq maladies ou signes de maladies infantiles.

\section{Méthodes}

\section{Collecte des données floristiques}

L'enquête ethnobotanique a été réalisée auprès de 708 personnes (hommes et femelles). L’approche utilisée a été l'entretien individuel et semi-direct avec des questionnaires. Les aspects abordés sont : le profil des enquêtés, les noms locaux des maladies traitées et les plantes médicinales utilisées ainsi que les recettes y afférant. L’identification taxonomique des échantillons récoltés a été réalisée à l’aide de la Flore Analytique du Bénin (Akoègninou et al., 2006) et par comparaison aux herbiers de référence disponibles à l’Herbier National du Bénin. Les différents échantillons ont été 
récoltés frais puis soigneusement lavés à l'eau de robinet puis à l'eau distillée et séchés à l'air libre à l'ombre au laboratoire pendant deux semaines. Ils ont été ensuite réduits en poudre à l'aide d'un broyeur électrique. La poudre obtenue est tamisée (mailles de 0,2 $\mathrm{mm}$ ) et stockée dans des boites étanches pour utilisation en cas de besoin pour les différents tests chimiques.

\section{Choix des maladies des enfants les plus traitées par la population}

Parmi les maladies des enfants recensées, cinq ont été choisies selon les critères ci-après : la prévalence élevée de la maladie (taux de citation supérieur à 5\%), l'appartenance à l'une des maladies prioritairement traitées dans les centres de santé au Bénin et être une maladie affectant les enfants quel que soit l'hygiène et les soins apportés à ces derniers.

\section{Choix des plantes prioritaires pour le traitement des maladies infantiles les plus citées}

Les espèces les plus connues et utilisées par les populations ont été déterminées selon la méthode proposée sur le groupe Tramil aux Caraïbes (Tramil 4, 1989) et utilisée par Deleke et al., (2011) et Fagbohoun, (2014). Cette méthode considère comme plantes à fréquence d'utilisation élevée, celles ayant été citées par au moins $20 \%$ des enquêtés.

\section{Préparation de l'extrait aqueux}

Pour préparer l'extrait aqueux, $5 \mathrm{~g}$ de la poudre sèche de chaque drogue ont été bouilli dans $50 \mathrm{ml}$ d'eau distillée, pendant 15 minutes. Le décocté a été filtré pour produire l'extrait aqueux.

\section{Criblage phytochimique}

Le criblage phytochimique qualitatif a été effectué sur des extraits aqueux en utilisant la méthode standard basée sur des réactions de coloration et/ou de précipitation comme décrites par Houghton et Raman (1998) et utilisée par Adjatin et al. (2013). Les analyses phytochimiques ont été effectuées dans le Laboratoire de Pharmacognosie et des huiles essentielles au Champ de Foire à Cotonou. Le tableau 1 indique les différents groupes chimiques recherchés et le principe des réactions. 
Tableau 1 : Test de mise en évidence et d'observation des métabolites secondaires ;

\begin{tabular}{|c|c|}
\hline Composés chimiques & Principe de la réaction \\
\hline Alcaloïdes & $\begin{array}{l}\text { En milieu acide et en présence du réactif de Mayer (solution aqueuse d'iodure double } \\
\text { de mercure et de potassium), les alcaloïdes se combinent avec le mercure et forment } \\
\text { un précipité blanc }\end{array}$ \\
\hline Tanins & $\begin{array}{l}\text { Avec les sels ferriques, les tanins donnent une coloration bleue ou noire ou un } \\
\text { précipité rose. }\end{array}$ \\
\hline Tanins Catéchiques & Avec le réactif de Stiasny, les tanins catéchiques donnent un précipité rose. \\
\hline Tanins Galliques & $\begin{array}{l}\text { En présence du chlorure ferrique et saturation avec l'acétate de sodium, les tanins } \\
\text { Galliques donnent une teinte bleue. }\end{array}$ \\
\hline Flavonoïdes & $\begin{array}{l}\text { En milieu acide et en présence de poudre de magnésium, les flavonoïdes donnent des } \\
\text { colorations spécifiques. Ainsi, les flavonols sont colorés en orangé et les flavanones } \\
\text { sont colorés en rouge (Réaction à la cyanidine). }\end{array}$ \\
\hline Anthocyanes & $\begin{array}{l}\text { En solution avec l'acide chlorhydrique et l'ammoniac à } 50 \% \text {, les anthocyanes se } \\
\text { colorent en rouge violacé }\end{array}$ \\
\hline Leuco-anthocyanes & $\begin{array}{l}\text { En présence de l’alcool chlorhydrique, les leuco-anthocyanes donnent une coloration } \\
\text { rouge brun (Réaction de Shinoda). }\end{array}$ \\
\hline Dérivés quinoniques & $\begin{array}{l}\text { En dissolvant les quinones en milieu alcalin aqueux, la solution prend une teinte vive } \\
\text { allant du rose au rouge violacée. Cette teinte varie selon la structure de la quinone } \\
\text { (naphtoquinone, anthraquinone) (Réaction de Bornträger). }\end{array}$ \\
\hline Saponosides & $\begin{array}{l}\text { Ils sont mis en évidence par l'indice de mousse qui est fourni par le degré de dilution } \\
\text { d'un décocté aqueux de la drogue qui, dans des conditions déterminées, donne une } \\
\text { mousse persistante. }\end{array}$ \\
\hline Triterpénoïdes & $\begin{array}{l}\text { En solution avec l'alcool éthylique et d'acétate de plomb, les triterpénoïdes se } \\
\text { colorent en violet, bleu ou verte (Réaction de Liebermann-Buchard). }\end{array}$ \\
\hline Stéroïdes & $\begin{array}{l}\text { En solution avec l'alcool éthylique et d'acétate de plomb, les stéroïdes donnent une } \\
\text { coloration rouge au vin (Réaction de Kedde). }\end{array}$ \\
\hline Mucilage & En présence de l'alcool absolu, les mucilages donnent un précipité floconneux \\
\hline Coumarines & $\begin{array}{l}\text { La présence de fluorescence suite à un test avec l'éther et l'ammoniac indique la } \\
\text { présence de coumarines. }\end{array}$ \\
\hline Composés réducteurs & $\begin{array}{l}\text { L’apparition d'un précipité rouge brique suite au test avec la liqueur de Fehling } \\
\text { indique la présence des sucres réducteurs. }\end{array}$ \\
\hline Anthracéniques libres & $\begin{array}{l}\text { En présence du chloroforme et de l'ammoniac, les anthracéniques libres ne donnent } \\
\text { pas de coloration rouge. }\end{array}$ \\
\hline $\begin{array}{l}\text { Anthracéniques } \\
\text { combinés O-hétérosides }\end{array}$ & $\begin{array}{l}\text { En milieu acide avec du chloroforme et de l'ammoniac, les O-hétérosides donnent } \\
\text { une coloration rouge. }\end{array}$ \\
\hline $\begin{array}{l}\text { Anthracéniques } \\
\text { combinés C-hétérosides }\end{array}$ & $\begin{array}{l}\text { En milieu ferrique avec du chloroforme et de l'ammoniac, les C-hétérosides donnent } \\
\text { une coloration rouge. }\end{array}$ \\
\hline $\begin{array}{l}\text { Hétérosides } \\
\text { cardiotoniques à } \\
\text { gemme cardénolide }\end{array}$ & $\begin{array}{l}\text { En milieu alcalin }(\mathrm{NaOH}) \text {, l'acide dinitrobenzoïque s'additionne sur la } \gamma \text { lactone } \alpha, \beta \text { - } \\
\text { insaturé du cardénolide pour former un dérivé fortement coloré en rouge pourpre ou } \\
\text { rouge au vin (Réaction de Kedde). }\end{array}$ \\
\hline $\begin{array}{l}\text { Dérivés } \\
\text { cyanogénétiques }\end{array}$ & Les ions cyanures réagissent avec l'acide picrique et donnent une coloration marron. \\
\hline
\end{tabular}

\section{Traitement des données}

Des calculs de fréquences ont été effectués pour diverses variables (plantes, maladies). Une espèce végétale est choisie pour l'étude phytochimique si elle respecte les critères de choix de plante fixés. 


\section{Résultats}

\section{Affections prioritaires infantiles et plantes médicinales associées}

$\mathrm{Au}$ total, 50 maladies et symptômes de maladies ont été cités et soignés par les populations (figure 1). En fonction des critères fixés, le paludisme, les candidoses, les troubles de la poussée dentaire, la colique du nourrisson et la diarrhée sont les affections infantiles les plus importantes pour les populations au sud du Bénin.

Le traitement de ces affections fait appel à dix (10) importantes plantes (tableau 2). Il s'agit de: Senna siamea et Dichapetalum madagascariensis pour le traitement du paludisme, Argemone mexicana et Caesalpinia bonduc utilisés pour contrôler les crises de la colique du nourrisson, Hyptis suaveolens et Ocimum gratissimum sont plus utilisés dans la prise en charge des candidoses, Clerodendrum volubile et Remirea maritima sont plus utilisées pour faciliter la poussée dentaire des nourrissons et enfin Psidium guajava et Combretum mucronatum pour le traitement de la diarrhée infantile. 


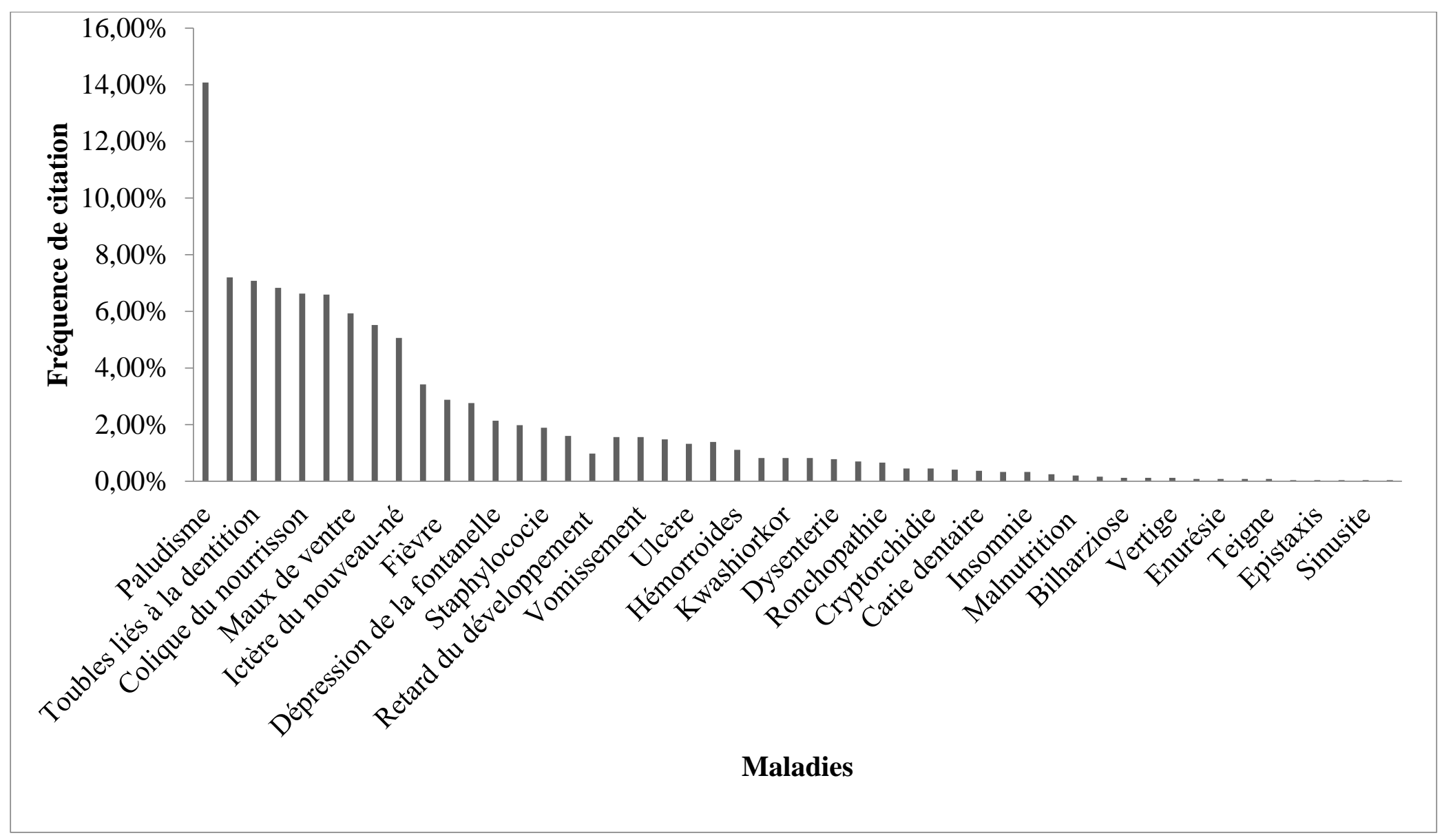

Figure 1: Fréquence de citation des maladies. 
Tableau 2 : Espèces végétales les plus utilisées dans le traitement des maladies infantiles prioritairement citées et traitées par les populations au sud du Bénin

\begin{tabular}{ccc}
\hline Maladies & Espèces végétales & Fréquence de recettes (Fr) \\
\hline Paludisme & Senna siamea (Lam.) H.S.Irwin & $11 \%$ \\
& \& Barneby & \\
& Dichapetalum & $4,90 \%$ \\
Colique du nourrisson & madagascariensis Poir. & \\
& Argemone mexicana L. & $23,47 \%$ \\
Candidose & Caesalpinia bonduc (L.) Roxb. & $12,25 \%$ \\
& Hyptis suaveolens (L.) Poit. & $11,25 \%$ \\
Troubles de la poussée dentaire & Ocimum gratissimum L. & $6,88 \%$ \\
& Clerodendrum volubileP. & $19,95 \%$ \\
& Beauv. & $9,75 \%$ \\
& Remirea maritima Aubl. & $78,94 \%$ \\
Diarrhée & Psidium guajava L. & $24,56 \%$ \\
& Combretum mucronatum & \\
\hline
\end{tabular}

\section{Criblage phytochimique}

L'analyse phytochimique des dix espèces végétales utilisées pour les soins de santé des enfants au sud du Bénin révèle que ces espèces sont constituées de différents groupes chimiques à savoir: les alcaloïdes, les tanins galliques et catéchiques, les coumarines, les anthocyanes, les leucoanthocyanes, les dérivés quinoniques, les triterpènoïdes, les stéroïdes, les saponosides et les composés réducteurs (tableau 3).

Aucune des plantes, à l'exception de Caesalpinia bonduc (qui contient les anthracéniques libres et les O-hétérosides), ne renferme les cardénolides, les dérivés cyanogéniques, les anthracéniques, les Ohétérosides et les C-hétérosides.

Les espèces Senna siamea et Dichapetalum madagascariense utilisées dans le traitement du paludisme infantile contiennent des alcaloïdes, des tanins galliques et catéchiques, des flavones, des anthocyanes, des dérivés quinoniques, des stéroïdes, des mucilages, des composés réducteurs. Mais Dichapetalum madagascariense contient en plus des leuco-anthocyanes, des triterpénoides et des coumarines.

Les plantes à savoir: Hyptis suaveolens et Ocimum gratissimum employées dans le traitement des candidoses contiennent des alcaloïdes, des tanins galliques et catéchiques, des anthocyanes, des dérivés quinoniques, des triterpénoides, des stéroïdes, des mucilages et des composés réducteurs. Ocimum gratissimum renferme en plus des flavones et des saponosides.

L'analyse des extraits aqueux de Clerodendrum volubile et de Remirea maritima utilisées pour le traitement de la dentition a révélé l'existence des tanins galliques et catéchiques, anthocyanes, leucoanthocyanes, dérivés quinoniques, saponosides, triterpénoïdes, stéroïdes et 
composés réducteurs. En plus de ces éléments, Clerodendrum volubile contient des alcaloïdes et des flavones.

Argemone mexicana et Caesalpinia bonduc utilisées dans le traitement de la colique du nourrisson renferment des alcaloïdes, des tanins galliques et catéchiques, des flavones, des dérivés quinones, des mucilages et des composés réducteurs. Mais Caesalpinia bonduc possède en plus des anthocyanes, des saponosides, des stéroïdes, des coumarines, des anthracéniques libres et des O-hétérosides tandis que Argemone mexicana possède des triterpénoides.

Quant aux plantes employées pour guérir la diarrhée infantile (Psidium guajava et Combretum mucronatum), elles renferment des tanins galliques et catéchiques, des anthocyanes, des dérivés quinoniques, des triterpénoides, des mucilages, des coumarines et des composés réducteurs. Mais dans Psidium guajava, on retrouve en plus de ces composés, les alcaloïdes, des flavones, des leuco-anthocyanes et les stéroïdes. 
Tableau 3 : Screening phytochimique dex dix (10) échantillons de plantes

+ : Présence; - : Absent.

Pa: Paludisme ; Ca : Candidose ; Co : Colique du nourrisson ; De : troubles de dentition ; Di : Diarrhée. Pa1: Senna siamea, Pa2 : Dichapetalum madagasriensis; Ca1: Hyptis suaveolens; Ca2: Ocimum gratissimum; Co1: Argemone mexicana; Co2 : Caesalpinia bonduc; De1: Clerodendrum volubile ; De2 : Remirea maritima ; Di1 : Psidium guajava ; Di2 : Combretum mucronatum; Al : Alcaloïdes ; Fl : Flavonoïdes ; Ta : Tallins ; Ga : Gallique ; Ca : Catéchique ; Cou : Coumarines ; Ant : Anthocyanes ; Leu : Leuco-anthocyanes ; Qui : Dérivés quinones ; Trit : Triterpénoïdes; Ste : Stéroïdes; Sa : Saponosides; Mu : Mucilages ; Ré : Composés réducteurs ; Car : Cardénolides ; Cya : Dérivés cyanogéniques ; O-h : O-hétérosides ; C-h : C-hétérosides ; Anth : Anthracéniques libres. Flav : flavone.

\begin{tabular}{|c|c|c|c|c|c|c|c|c|c|c|c|c|c|c|c|c|c|c|c|}
\hline \multirow{4}{*}{$\begin{array}{c}\text { Maladies } \\
\text { traitées }\end{array}$} & \multirow[t]{4}{*}{ Plantes } & \multicolumn{18}{|c|}{ Groupes chimiques } \\
\hline & & \multirow[t]{3}{*}{ Al } & \multicolumn{7}{|c|}{ Composés phénoliques et les phénols } & \multirow[t]{3}{*}{ Trit } & \multirow[t]{3}{*}{ Ste } & \multirow[t]{3}{*}{ Sap } & \multirow[t]{3}{*}{$\mathbf{M u}$} & \multirow[t]{3}{*}{$\mathbf{R e}$} & \multicolumn{5}{|c|}{ Hétérosides } \\
\hline & & & \multirow[t]{2}{*}{ Fl } & \multicolumn{2}{|c|}{ Ta } & \multirow[t]{2}{*}{ Cou } & \multirow[t]{2}{*}{ Ant } & \multirow[t]{2}{*}{ Leu } & \multirow{2}{*}{ Qui } & & & & & & \multirow[t]{2}{*}{ Car } & \multirow[t]{2}{*}{ Суа } & \multirow[t]{2}{*}{ O-h } & \multirow[t]{2}{*}{ C-h } & \multirow{2}{*}{$\begin{array}{c}\mathbf{A n} \\
\mathbf{h}\end{array}$} \\
\hline & & & & $\mathrm{Ga}$ & $\mathrm{Ca}$ & & & & & & & & & & & & & & \\
\hline \multirow[t]{2}{*}{$\mathrm{Pa}$} & Pa1 & + & flav & + & + & - & + & - & + & - & + & - & + & + & - & - & - & - & - \\
\hline & $\mathrm{Pa} 2$ & + & Flav & + & + & + & + & + & + & + & + & - & + & + & - & - & - & - & - \\
\hline \multirow[t]{2}{*}{$\mathrm{Ca}$} & Ca1 & + & - & + & + & - & + & - & + & + & + & - & + & + & - & - & - & - & - \\
\hline & $\mathrm{Ca} 2$ & + & Flav & + & + & - & + & + & + & + & + & + & + & + & - & - & - & - & - \\
\hline \multirow[t]{2}{*}{ Co } & Co1 & + & Flav & + & - & - & - & + & + & + & - & - & + & + & - & - & - & - & - \\
\hline & Co2 & + & Flav & + & + & + & + & - & + & - & + & + & + & + & - & - & + & - & + \\
\hline \multirow[t]{2}{*}{$\mathrm{De}$} & De1 & + & Flav & + & + & - & + & + & + & + & + & + & - & + & - & - & - & - & - \\
\hline & De2 & - & - & + & + & - & + & + & + & + & + & + & - & + & - & - & - & - & - \\
\hline \multirow[t]{2}{*}{$\mathrm{Di}$} & Di1 & + & Flav & + & + & + & + & + & + & + & + & - & + & + & - & - & - & - & - \\
\hline & Di2 & - & - & + & + & + & + & - & + & + & - & - & + & + & - & - & - & - & - \\
\hline
\end{tabular}




\section{Discussion}

L’importante diversité de la flore béninoise en plantes médicinales n’est plus à démontrer. Les études conduites par Adjanohoun et al. (1989) et celles réalisées par Dassou et al. (2015) ont rapporté respectivement 507 plantes utilisées dans le traitement des maladies et symptômes de maladies humains et 241 plantes à usages vétérinaires. La valorisation de ces plantes passe par des études phytochimiques, pharmacologiques et cliniques pour des perspectives de formulation de Médicaments Traditionnellement Améliorés. Le présent travail rentre dans cette dynamique et a montré l'usage de 10 plantes prioritaires dans le traitement des pathologies et signes cliniques infantiles. Les recherches phytochimiques réalisées ont montré une diversité de métabolites secondaires susceptibles de révéler ou de prouver l'usage de ces plantes en médecine infantile. C'est le cas des alcaloïdes présents dans toutes les plantes à l'exception de Remirea maritima et Combretum mucronatum. Selon Badiaga (2011), ce groupe chimique est très recherché pour son large spectre d'activités biologiques dont les propriétés anti malariques et antibiotiques ainsi que des propriétés antalgiques et spasmolytiques (Charpentier et al., 2008). Les alcaloïdes constitueraient donc l'un des métabolites recherchés par la population pour le traitement du paludisme et de la diarrhée et même pour le contrôle de la colique du nouveau-né.

Les composés phénoliques et les phénols (flavonoïdes, tanins, coumarines, anthocyanes, leuco-anthocyanes, quinones) présents dans les plantes sont des antioxydants naturels qui sont selon Min et Ebeler (2008) des composés capables d'interférer avec les radicaux libres générés en permanence par l'organisme humain ou formés en réponse à des agressions de notre environnement (polluants, infections...). Parmi eux, les flavonoïdes possèdent une forte activité antiparasitaire sur un large spectre de parasites comme le genre Plamodium (falciparum) et manifestent des activités antispasmodiques (Portet et al., 2007), ce qui pourrait expliquer leur usage dans le traitement du paludisme et de la colique du nourrisson. Les flavonoïdes et coumarines possèdent des propriétés anti-inflammatoires, et antiseptiques (Igor, 2002), antifongique et antibactériennes (Kuster et al., 2009) et pourraient justifier leur usage dans le traitement de la candidose chez les enfants. Aussi, les terpénoïdes montrent-ils d'excellentes activités antibactériennes contre diverses bactéries ainsi que Candida albicans qui constitue l'agent causal de la candidose (Fagbohun et al., 2012) et expliquerait leur utilisation dans le traitement de cette affection.

Les coumarines manifestent également des activités analgésique, antioxydant et antimicrobienne et sont efficaces contre les bactéries Gram positifs (Marquis et al., 2012). Elles seraient pour cette raison utilisées dans le traitement du paludisme, de la colique du nourrisson et de la diarrhée chez 
l'enfant. Par contre, d'autres comme les tanins montrent d'excellentes activités antiparasitaires (Brunet et Hoste, 2006) et peuvent être utilisés pour limiter les secrétions excessives comme la diarrhée. Par ailleurs, les tanins ayant également pour effet de réduire l'irritabilité et la douleur, sont employés le plus souvent contre les inflammations de la cavité buccale, les inflammations dermiques (Vijayameena et al., 2013) générées par la poussée dentaire chez les nourrissons. Les tanins sont aussi capables de former une couche de coagulation isolante et protectrice des muqueuses et des tissus et contribueraient au traitement de la colique du nourrisson. Les autres symptômes (irritations des gencives boursouflées et rouges, douleur, rougeurs cutanées, diarrhée, rhume et fièvre) qui accompagnent la poussée dentaire sont traités par les terpénoïdes, les stéroïdes et les saponosides qui sont de puissants antioxydants, antalgiques, antimicrobiennes (Vincken et al., 2007 ; Yamunadevi et al., 2011).

Aucune des plantes étudiées ne renferme les cardénolides, les dérivés cyanogéniques, et les C-hétérosides. Les dérivés cyanogéniques sont de véritables poisons métabolites et leur absence dans ces plantes peut rassurer les consommateurs des risques liés à leur consommation. Les cardénolides sont des substances naturelles qui agissent sur le cœur en régularisant les contractions cardiaques sans augmenter la quantité d'oxygène dans les muscles cardiaques (Adjatin et al. 2013). Cependant, ces composés pourraient devenir de véritables poisons en bloquant la relaxation pendant la diastole puisque la marge est très étroite entre la dose thérapeutique et la dose toxique. Par conséquent, une forte dose pourrait être à l'origine d'un arrêt cardiaque. En tenant compte de la fragilité de l'organisme des enfants, l'absence de ces composés chimiques serait souhaitable.

De ces analyses, on pourrait déduire une certaine compatibilité entre les propriétés des métabolites trouvés et les objectifs thérapeutiques recherchés dans les traitements traditionnels des maladies infantiles.

\section{Conclusion}

Au Sud-Bénin, la pharmacopée est encore déterminante dans le traitement des maladies infantiles. Au total, 10 plantes prioritaires sont utilisées pour traiter les 5 pathologies et signes cliniques infantiles les plus fréquentes. Le criblage phytochimique de ces plantes a permis d'identifier des alcaloïdes, des tanins (gallique et catéchique), des coumarines, des anthocyanes et leuco-anthocyanes, des dérivés quinones, des triterpénoïdes, des stéroïdes, des saponosides et des sucres réducteurs qui constituent une base scientifique de l'utilisation thérapeutique de ces plantes étudiées. Ces métabolites secondaires possèdent diverses propriétés qui expliqueraient leur usage par les populations pour le traitement des affections indiquées. Cependant l'évaluation des activités antibactériennes, analgésiques, et autres 
de même que le test de toxicité de l’extrait aqueux de ces plantes sont nécessaires.

\section{References:}

1.Adjanohoun, E.J., Adjakidje V., Ahyi M.R.A., Aké Assi L., Akoegninou A., D’Almeida J., Apovo F., Boukef K., Chadare M., Cusset G., Dramane K., Eymé J., Gassita J.N., Gbaguidi N., Goudoté E., Guinko S., Houngnon P., Lo I., Keita A., Kiniffo H.V. 1989. Contribution aux études ethnobotaniques et floristiques en République Populaire du Bénin. Paris : ACCT, « Médecine traditionnelle et pharmacopée », 895 p.

2. Adjatin A., Dansi A, Badoussi E, Loko Y. L., Dansi M., Gbaguidi F, Azokpota P, Ahissou H., Akoègninou A., Akpagana K. and Sanni A. 2013. Phytochemical screening and toxicity studies of Crassocephalum rubens (Juss. ex Jacq.) S. Moore and Crassocephalum crepidioides (Benth.) S. Moore consumed as vegetable in Benin. Journal of Chemical and Pharmaceutical Research, , 5(6):160-167.

3. Adomou A.C., Agbani O.P., Sinsin B. 2011. Plantes. In Protection de la Nature en Afrique de l'Ouest: Une Liste Rouge pour le Bénin. Nature Conservation in West Africa: Red List for Benin, Neuenschwander P, Sinsin B, Goergen G (eds). International Institute of Tropical Agriculture: Ibadan, Nigeria, 21-46.

4. Adomou A.C., Yedomonhan H, Djossa B, Legba. I., M. Oumorou M. et Akoegninou A. 2012. Etude Ethnobotanique des plantes médicinales vendues dans le marché d’Abomey-Calavi au Bénin. Int. J. Biol. Chem. Sci. 6, 745-772.

5. Agbankpé A. J., Bankolé S. H., Assogba F., Dougnon T. V., Yèhouénou B., Gbénou J. and Baba-Moussa L. 2015. Phytochemical Screening and Cytotoxic Analysis of Three Local Vegetables Used in the Treatment of Bacterial Diarrhoea in Southern Benin (West Africa): A Comparative Study. BBJ, 9(4): 1-13, Article no.BBJ.19123

6. Akoègninou A. 2004. Recherches botaniques et écologiques sur les forêts actuelles du Bénin. Thèse d'Etat. Université de CocodyAbidjan (Côte d'Ivoire). 326p.

7. Akoègninou $\mathrm{A}$, van der Burg WJ, van der Maesen L J G. 2006. Flore Analytique du Bénin. Backhuys Publishers: Wageningen; 1034p.

8. Anonyme, 1974. Encyclopédie-Le Grand Médical. L’histoire de la médecine et de la chirurgie, l'avenir de la médecine, les prix Nobel. Edition Service S.A., Genève (Suisse), 397 pp. 
9. Badiaga M., 2011. Etude ethnobotanique, phytochimique et activités biologiques de Nauclea latifolia Smith, une plante médicinale africaine récoltées au Mali. Université de Bamako, p136 + Annexe.

10. Brunet S. et Hoste H. 2006. Les monomères des tanins condensés affectent le dégainement des larves des nématodes parasites des ruminants. Journal of Agricultural and Food Chemistry, 54: 74817487.

11. Charpentier B., Hamon-Lorleach F., Harlay A., Huard A., Ridoux L., Chancellé S., 2008. Guide du préparateur en pharmacie, Ed. Masson, 3eme édition, 1123.

12. Dassou G. H., Adomou A.C., Yédomonhan H., Ogni C. A., Tossou M., Dougnon J. T. et Akoègninou A. 2015. Flore médicinale utilisée dans le traitement des maladies et symptômes animaux au Bénin. Journal of Animal \& Plant Sciences, 26 (1) : 4036-4057.

13. Deleke I.K. E., Djego J., Gbenou J., Hounzangbe-Adote S.M., et Sinsin B. 2011. Etude phytochimique des principales plantes galactogènes et emménagogues utilisées dans les terroirs riverains de la Zone cynégétique de la Pendjari. Int. J. Biol. Chem. Sci. 5(2): 618633.

14. Dresse A., De Baeremaeker D. 2013. Amawato Le marché de la santé au pays du vaudou (C) CTB, Bruxelles, août 2013 www.btcctb.org.

15. Fagbohun E. D., Lawal, O. U. and Ore, M. E. 2012. The proximate, mineral and phytochemical analysis of the leaves of Ocimum gratissimum L., Melanthera scandens A. and Leea guineensis L. and their medicinal value. International Journal of Applied Biology and Pharmaceutical Technology Volume: 3: ISSN 0976-4550

16. Houghton P.J., Raman A. 1998. Laboratory Handbook for the Fractionation of Natural Extracts. Chapman and Hall, New York, 5: 103-108.

17. Igor Passi L B. (2002). Etude des activités biologiques de Fagara zanthoxylo_des Lam. (Rutaceae). Thèse Pharmacie, Bamako ; $133 \mathrm{P}$

18. Igue AM., Saidou A., Adjanohoun A., Ezui G., Attiogbe P., Kpagbin G., Gotoechan- Hodonou H., Youl S., Pare T., Balogoun I., Ouedraogo J., Dossa E., Mando A.et Sogbedji JM. Evaluation de la fertilité des sols au sud et centre du Bénin. Bulletin de la Recherche Agronomique du Bénin (BRAB).), 1840-7099.

19. INSAE (Institut National de la Statistique et de l'Analyse économique). 2013. Recensement Général de la Population et de l'Habitat. Résultats Provisoires du RGPH4, MDAEP. INSAE: Cotonou, Bénin; 8p.

20. Kolling M., Winkley K. et Von Deden M. 2010. “For someone who’s rich, it's not a problem.” Insights from Tanzania on diabetes health- 
seeking and medical pluralism among Dar es Salam's urban poor. Globalization and Health, 6:8.

21. Kouchadé A. S., Adomou A. C., Tossou G. M., Yédomonhan H., Dassou G. H. et Akoègninou A. 2016. Étude ethnobotanique des plantes médicinales utilisées dans le traitement des maladies infantiles et vendues sur les marchés au sud du Bénin. Journal of Animal \&Plant Sciences. Vol.28, Issue 2: 4418-4438.

22. Kuster R. Arnold N., Wessjohann L. 2009. Anti-fungal flavonoids from Tibouchina grandifolia. Biochem. Syst. Ecol., 37(1) : 63-65.

23. Lagnika 1. 2005. Etude phytochimique et activité biologique de substances naturelles isolées de plantes béninoises. Université Louis pasteur Strasbourg en cotutelle avec l’Universit2 d'Abomey-Calavi Bénin.

24. Mangambu M., Kamabu V., Bola M.F. 2008. Les plantes médicinales utilisées dans le traitement de l'asthme à Kisangani et ses environs (Province Orientale, R.D.Congo). Annales des Sciences. Université Officielle de Bukavu, 1 (1) : 63-68.

25. Mangambu M., Van Diggelen R Mwanga Mwanga, J-C., Ntahobavuka H. Malaisse F. \& Robbrecht E. 2012. Etude ethnoptéridologique, évaluation des risques d'extinction et stratégies de conservation aux alentours du Parc National de Kahuzi Biega en R.D. Congo. Geo-Eco-Trop, 36 (1/2) : 137-15.

26. Mangambu M., 2013. Taxonomie, biogéographie et écologie des Ptéridophytes de l'écosystème forestier des montagnes du Parc National de Kahuzi-Biega à l'Est de la R.D. Congo. Thèse de doctorat, Université d'Anvers/Belgique, 463 p.

27. Mangambu M.J.D., Mushagalusa K. F., Kadima N. J. 2014. Contribution à l'étude phytochimique de quelques plantes médicinales antidiabétiques de la ville de Bukavu et ses environs (Sud-Kivu, R.D.Congo). Journal of Applied Biosciences 75:62116220.

28. Marquis A., 2012. Propriétés antibactérienne, anti adhérence, antiinflammatoire et anti-protéase de deux coumarines, l'Auraptène et le Lacinartin. Thèse de doctorat. Université Laval Québec.

29. Min, K., Ebeler, S.E. 2008. Flavonoid effects on DNA oxidation at low concentrations relevant to physiological levels. Food and Chemical Toxicology, 46: 96-10.

30. MS/PNPMT, 2009. Ministère de la Santé/ Programme National de la Pharmacopée et de la Médecine Traditionnelles, 2009. La pharmacopée et la médicine traditionnelle au Bénin : état des lieux et perspectives. $14 \mathrm{p}$.. 
31. N’guessan K., Kadja B., Zirihi G. N., Traoré D. \& Aké-Assi L. 2009. Screening phytochimique de quelques plantes médicinales ivoiriennes utilisées en pays Krobou (Agboville, Côte-d'Ivoire) Sciences \& Nature Vol. $6 N^{\circ} 1: 1-15$

32. OMS (Organisation Mondiale de la Santé) 2002. Stratégie de l’OMS pour la médecine traditionnelle pour 2002-2005. WHO/EDM/TRM/2002, Genève: 65.

33. OMS. 2012. Stratégie pour la médecine traditionnelle 2007-2011. Genève.

34. Portet, B., Fabre, N., Roumy, V., Gornitzka, H., Bourdy, G., Chevalley, S., Sauvain, M., Valentin, A., Moulis, C., 2007. Activityguided isolation of antiplasmodial dihydrochalcones and flavanones from Piper hostmannianum var. berbicense. Phytochemistry 68 13121320.

35. Shuaibu MN, Wuyep PA, Yanagi T, Hirayama K, Tanaka T. et Kouno I : 2008. The use of microfluorometric method for activityguided isolation of antiplasmodial compound from plant extracts. Parasitology Research 102:1119-1127.

36. Singh B. \& Singh BK, 2012. Ethnomedicinal use of Pteridophytes in reproductive health of tribal women of Pachmarhi Biosphere Reserve, Madhya Pradesh, India. International Journal of Medicine and Medical researcher, 3, 12: 4780-4790.

37. SNIGS-MS (Système National d'Information et de Gestion SanitaireMinistère de la Santé), 2014. Annuaire des statistiques sanitaires 2013, 151p.

38. Soro D, Koné MW. et Kamanzi Atindehou K: 2010. Evaluation des Activités Antimicrobiennes et Anti-Radicaux Libres de Quelques Taxons Bioactifs de Côte d'Ivoire. European Journal of Scientific Research 40 (2): 307-317.

39. Tramil 4 1989. Vers une pharmacopée aux Caraïbes. Recherche Scientifique et Usage Populaire des Plantes Médicinales dans le Caraïbe. Robineau L (éd) : 234-267.

40. Tra Bi F.H., Koné M.W. et Kouamé N.F. Antifungal activity of Erigeron floribundus (Asteraceae) from Côte d'Ivoire, West Africa. Tropical Journal of Pharmaceutical Research 7 (2): 975-979, 2008.

41. Vanhaelen M. 2002. Evolutions potentielles de l'héritage phytothérapeutique traditionnel. SOMA, $1: 73-81$.

42. Vijayameena C, Subhashini G, Loganayagi M, Ramesh B. 2013. Phytochemical screening and assessment of antibacterial activity for the bioactive compounds in Annona muricata. Int. Curr. Microbiol. App. Sci. 2(1):1-8. 
43. Vincken, J.P., Heng, L., De Groot, A., Gruppen, H. 2007. Review Saponins, classification and occurrence in the plant kingdom. Phytochemistry 68, 275-297

44. Yamunadevi M., Wesely E.G., Johnson M. 2011. Phytochemical studies on the terpenoids of medicinally imporat plant Aerva lanata L. using HPTLC. A. Pacific J. of Trop.Biomedicine. S220-S225.

45. Zerbo P., Millogo-Rasolodimby J., Nacoulma-Ouedraogo O. G., Damme P. V. 2007. Contribution à la connaissance des plantes médicinales utilisées dans les soins infantiles en pays San, au Burkina Faso. Int. J. Biol. Chem. Sci., 1, 262-274. 